\title{
MICROSPHERE A DRUG DELIVERY SYSTEM-A REVIEW
}

\author{
MANOJ KUMAR DAS*, ABDUL BAQUEE AHMED, DIPANKAR SAHA
}

Girijananda Chowdhury Institute of Pharmaceutical Science (GIPS), Hatkhowapara, Azara, Guwahati 781017 Assam Email: mdas5416@gmail.com

Received: 15 Apr 2019, Revised and Accepted: 26 Jun 2019

\begin{abstract}
The targeted drug delivery is designed for endeavoring to concentrate the drug in the tissues of curiosity while reducing relative concentration of medication in the remaining tissues. There for drug is localized on the targeted site. Hence, surrounding tissues are not affected by the drug. Controlled drug delivery system can overcome the problems of conventional drug therapy and gives better therapeutic efficacy of a drug. Microspheres are characteristically free flowing powders consisting of proteins or synthetic polymers having a particle size ranging from 1-1000 $\mu \mathrm{m}$. The range of Techniques for the preparation of microspheres offers a Variety of opportunities to control aspects of drug administration and enhance the therapeutic efficacy of a given drug. There are various approaches in delivering a therapeutic substance to the target site in a sustained controlled release fashion. Microspheres has a drug located centrally within the particle, where it is encased within a unique polymeric membrane. In future various other strategies, microspheres will find the central place in novel drug delivery, particularly in diseased cell sorting, diagnostics, gene and genetic materials, safe, targeted and effective in vivo delivery and supplements as miniature versions of diseased organ and tissues in the body.
\end{abstract}

Keywords: Microspheres, Controlled release, Therapeutic efficacy, Novel drug delivery

(C) 2019 The Authors. Published by Innovare Academic Sciences Pvt Ltd. This is an open access article under the CC BY license (http://creativecommons.org/licenses/by/4.0/) DOI: http://dx.doi.org/10.22159/ijcpr.2019v11i4.34941

\section{INTRODUCTION}

Microspheres are small spherical particles, with diameters $10 \mu \mathrm{m}$ to $1000 \mu \mathrm{m}$. Microsphere play an important role to improve bioavailability of conventional drugs and minimizing side effects. The main advantage of applying microspheres as drugs delivery system is the controlled release of the drug content. Microencapsulation used for retarding the drug release from dosage forms and reduced the adverse effects, increased the patient for compliance. In this technique, aqueous insoluble core (drugs) coated with an aqueous insoluble coat (polymer) by emulsion solvent diffusion evaporation technique for sustain release drug delivery system. Microspheres can be made by a variety of ways including emulsification technique with single or double solvent evaporation system spray-dry technique or phase separation technique. Microspheres can be prepared by dissolving the starting materials in volatile solvents and then dispersing them in another solvent which is not miscible with the previous. Later complete evaporation of the last solvent will produce a fine powder called microspheres which is soluble in water. There are two types of microspheres;

- Microcapsules.

- Micrometrics.

Microcapsules are those in which entrapped substance is distinctly surrounded by distinct capsule wall and micrometrics in which entrapped substance is dispersing throughout the microspheres matrix. Solid biodegradable microspheres incorporating a drug dispersed or dissolved through particle matrix have the potential for the controlled release of drug. They are made up of polymeric, waxy, or other protective materials, that is, biodegradable synthetic polymers and modified natural products [1-3].

\section{Types of microspheres}

\section{Bioadhesive microspheres}

Adhesion can be defined as sticking of drug to the membrane by using the sticking property of the water soluble polymers. Adhesion of drug delivery device to the mucosal membrane such as buccal, ocular, rectal, nasal etc. can be termed as bio adhesion. These kinds of microspheres exhibit a prolonged residence time at the site of application and causes intimate contact with the absorption site and produces better therapeutic action. Carrier technology offers an intelligent approach for drug delivery by coupling the drug to a carrier particle such as microspheres, Nano spheres, liposomes, nanoparticles, etc., which modulates the release and absorption of the drug. Microspheres constitute an important part of these particulate drug delivery systems by virtue of their small size and efficient carrier capacity [4-6].

\section{Magnetic microspheres}

This kind of delivery system is very much important which localizes the drug to the disease site. In this larger amount of freely circulating drug can be replaced by smaller amount of magnetically targeted drug. Magnetic carriers receive magnetic responses to a magnetic field from incorporated materials that are used for magnetic microspheres are chitosan, dextran etc. The different types are therapeutic magnetic microspheres and diagnostic microspheres [7-9].

\section{- Therapeutic magnetic microspheres}

It is used to deliver chemotherapeutic agent to liver tumor. Drugs like proteins and peptides can also be targeted through this system.

\section{- Diagnostic microspheres}

It can be used for imaging liver metastases and also can be used to distinguish bowel loops from other abdominal structures by forming Nano size particles supramagnetic iron oxides.

\section{Floating microspheres}

In floating types the bulk density is less than the gastric fluid and so remains buoyant in stomach without affecting gastric emptying rate. The drug is released slowly at the desired rate, if the system is floating on gastric content, increases gastric residence and fluctuation in plasma concentration. It also reduces chances of striking and dose dumping and produces prolonged therapeutic effect. One another way it produces prolonged therapeutic effect and therefore reduces dosing frequencies $[10,11]$

\section{Polymeric microspheres}

The different types of polymeric microspheres can be classified as follows and they are biodegradable polymeric microspheres and synthetic polymeric microspheres.

\section{- Biodegradable polymeric microspheres}

Natural polymers such as starch are used with the concept that they are biodegradable, biocompatible, and also Bio adhesive in nature. 
Biodegradable polymers prolongs the residence time when contact with mucous membrane due to its high degree of swelling property with aqueous medium, results gel formation. The rate and extent of drug release is controlled by concentration of polymer and the release pattern in a sustained manner. The main drawback is, in clinical use drug loading efficiency of biodegradable microspheres is complex and is difficult to control the drug release.

\section{- Synthetic polymeric microspheres}

The interest of synthetic polymeric microspheres are widely used in clinical application, moreover that also used as bulking agent, fillers, embolic particles, drug delivery vehicles etc. and proved to be safe and biocompatible. But the main disadvantage of these kind of microspheres, are tend to migrate away from injection site and lead to potential risk embolism and further organ damage.

\section{Radioactive microspheres}

Radio embolization therapy microspheres sized 10-30 nm are of larger than capillaries and gets tapped in first capillary bed when they come across. They are injected to the arteries that lead to tumor of interest. So these radioactive microspheres deliver high radiation dose to the targeted areas without damaging the normal surrounding tissues. It differs from drug delivery system, as radio activity is not released from microspheres but acts from within a radioisotope typical distance and the different kinds of radioactive microspheres are $\alpha$ emitters, $\beta$ emitters, $\gamma$ emitters.

\section{Mucoadhesive microspheres}

Mucoadhesive microspheres which are of 1-1000 mm in diameter and consisting either entirely of a mucoadhesive polymer or having an outer coating of it and coupling of mucoadhesive properties to microspheres has additional advantages, $e . g$. efficient absorption and enhanced bioavailability of the drugs due to a high surface to volume ratio, a much more intimate contact with the mucus layer, specific targeting of drug to the absorption site achieved by anchoring plant lectins, bacterial adhesions and antibodies, etc. on the surface of the microspheres. Mucoadhesive microspheres can be tailored to adhere to any mucosal tissue including those found in eye, nasal cavity, urinary and gastrointestinal tract, thus offering the possibilities of localized as well as systemic controlled release of drugs [12].

\section{Advantages of microspheres over conventional dosage forms}

Microspheres provide prolonged and constant therapeutic effect. Particle size reduction for enhancing solubility of the poorly soluble drug. Microspheres reduce the dosing frequency and therefore improve the patient compliance. Protect the drug from enzymatic and photolytic cleavage hence found to be best for drug delivery of protein. Microspheres provide controlled, sustained and targeted delivery of the drug. Biodegradable microspheres have the advantage over large polymer implants in that they do not require surgical procedures for implantation and removal. Microspheres produce more reproducible drug absorption. Drug discharge in stomach is hindered and that's why local unwanted effects are reduced. In case of microspheres, better therapeutic effect for short half-life of drugs can be achieved. Microspheres provide freedom from drug and recipients incompatibilities especially with buffer. Microspheres reduce dose dumping. Microspheres provide the protection of drugs against environment. Microspheres also mask the taste and odor. Microspheres avoids the first pass metabolism. Microspheres can be easily injected in body because of their small and spherical size. Microspheres enhance the biological half-life and also improve the bioavailability. Controlled release delivery biodegradable microspheres are used to control drug release rates thereby decreasing toxic side effects, and eliminating the inconvenience of repeated injections. Microspheres also reduce the chances of G. I. irritation.

\section{Limitation}

The costs of the materials and processing of the controlled release preparation, are substantially higher than those of standard formulations. Controlled release rate of microspheres may vary due to certain factors like intrinsic or extrinsic factors may be food, rate of transit through gut, mucin turnover rate etc. The fate of polymer matrix and its effect on the environment. There are differences in release from one to another dosage form. Any loss of integrity in release pattern may cause potential toxicity. The fate of polymer additives such as plasticizers, stabilizers, antioxidants and filers. Reproducibility is less. Low drug loading is done in case of parental microspheres. Process situations like change in temperature, $\mathrm{pH}$, solvent addition, and evaporation/agitation may influence the stability of core particles to be encapsulated. Parental delivery of microspheres may interact or form complex with blood components. The environmental impact of the degradation products of the polymer matrix produced in response to heat, hydrolysis, oxidation, solar radiation or biological agents

\section{Method of preparation of microspheres}

\section{Single emulsion technique}

Several Carbohydrates and Proteins are mainly prepared by this technique. In this technique, natural polymers are first dissolved in aqueous medium and then dispersed in non-aqueous medium (oil phase) followed with next step crosslinking of dispersed globule; which can be achieved by 2 methods:

- By Heat: Addition of dispersion into heated oil, but this method is not suitable for thermo labile drugs.

- By Chemical Cross-linking Agent: Using glutaraldehyde, formaldehyde, acid chloride etc. as cross-linking agent. Chemical cross-linking suffers the disadvantage of excessive exposure

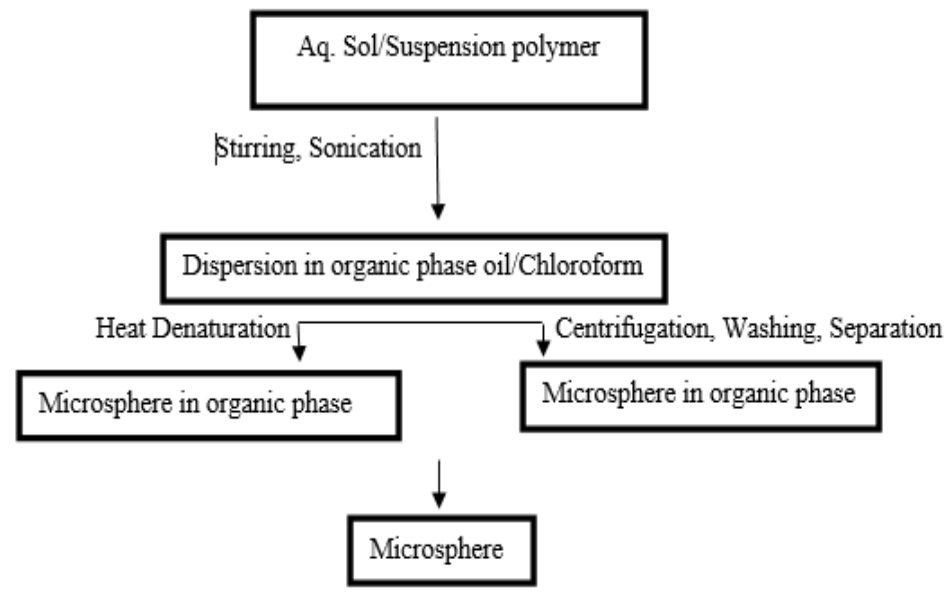

Fig. 1: Single emulsion technique 


\section{Double emulsion technique}

Double emulsion method of microspheres preparation involves the formation of the multiple emulsions or the double emulsion of type $\mathrm{w} / \mathrm{o} / \mathrm{w}$ and is best suited for water soluble drugs, peptides, proteins and the vaccines. This method can be used with both the natural as well as synthetic polymers. The aqueous protein solution is dispersed in a lipophilic organic continuous phase. The protein solution may contain the active constituents. The continuous phase is generally consisted of the polymer solution that eventually encapsulates of the protein contained in dispersed aqueous phase. The primary emulsion is exposed then to the homogenization or the sonication before addition to the aqueous solution of the poly vinyl alcohol (PVA). The results in the formation of a double emulsion. The emulsion is then subjected to solvent removal either by solvent evaporation or by solvent extraction. A number of hydrophilic drugs like luteinizing hormone releasing hormone (LH-RH) agonist, vaccines, proteins/peptides and conventional molecules are successfully incorporated into the microspheres using the method of double emulsion solvent evaporation/extraction.

Double Emuision

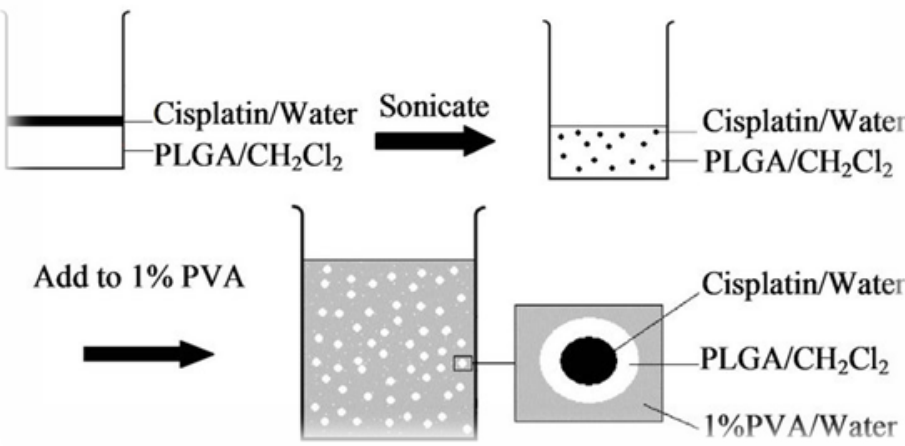

Fig. 2: Double emulsion technique

\section{Polymerization techniques}

The polymerization techniques conventionally used for preparing the microspheres are mainly classified as:

\section{- Normal polymerization}

- Interfacial polymerization.

Both are carried out in liquid phase.

- Normal polymerization: It is carried out by using different techniques as bulk, suspension, precipitation, emulsion and micellar polymerization methods. In bulk, a monomer or a combination of monomers along with the initiator or catalyst is usually heated to initiate polymerization. Polymer so obtained may be moulded as microspheres. Drug loading may be done during the polymerization process. Suspension polymerization also referred as bead or pearl polymerization. It is carried out by heating the monomer or composition of monomers as droplets dispersion in a continuous aqueous phase. Droplets may also contain an initiator and other additives. Emulsion polymerization deviates from suspension polymerization as due to the presence initiator in the aqueous phase, which afterwards diffuses to the surface of micelles. Bulk polymerization has merits of formation of pure polymers.

- Interfacial polymerization: This involves the reaction of various monomers at the interface between the two immiscible liquids to form a film of polymer that essentially envelops the dispersed phase.

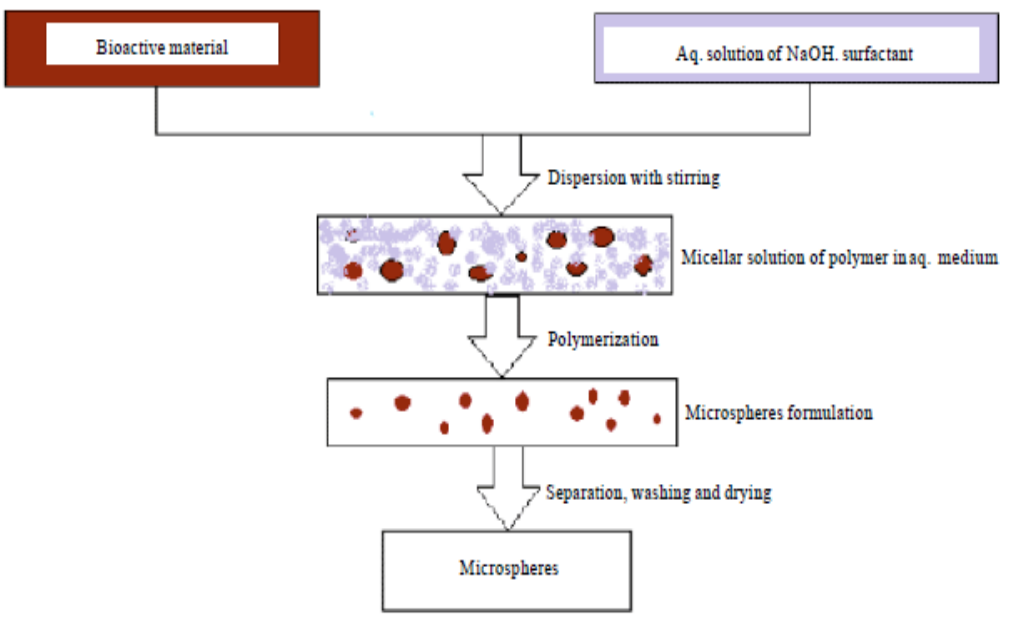

Fig. 3: Polymerization techniques

\section{Spray drying technique}

In this technique, the polymer is dissolved in volatile organic solvent like dichloromethane, acetone etc. and then drug (solid form) is dispersed in polymer solution under high speed homogenization. Dispersion is then atomized in the hot air stream, and atomization lead to the formation of small droplets from which solvent evaporates instantaneously; leading to formation of microsphere in a size range of 1-100 $\mu \mathrm{m}$. Prepared micro particles are separated by hot air by the help of cyclone separator and solvent traces is removed by vacuum drying $[13,14]$. 


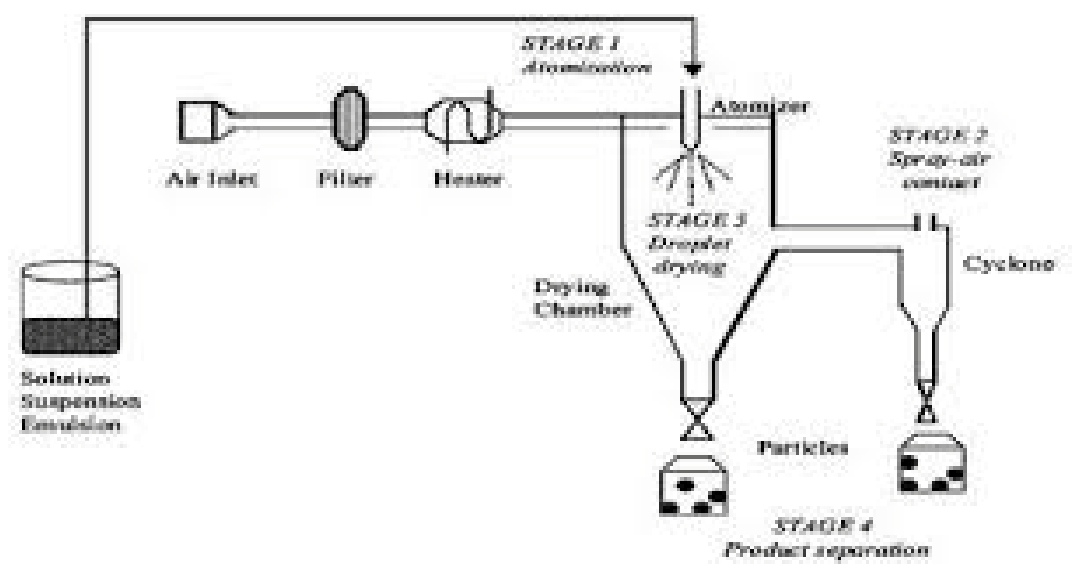

Fig. 4: Spray drying technique

\section{Solvent extraction}

Solvent evaporation method is used for manufacturing of micro particles, involves removal of the organic phase by extraction of the aqueous or non-aqueous solvent. This method involves water miscible organic solvents as isopropanol. Organic phase can be removed by extraction with water. This process decreases the hardening time for the microspheres. One variation of the process involves direct incorporation of the drug or protein to polymer organic solution. Rate of solvent removal by extraction method depends on the temperature of water, ratio of emulsion volume to the water and solubility profile of polymer $[15,16]$.

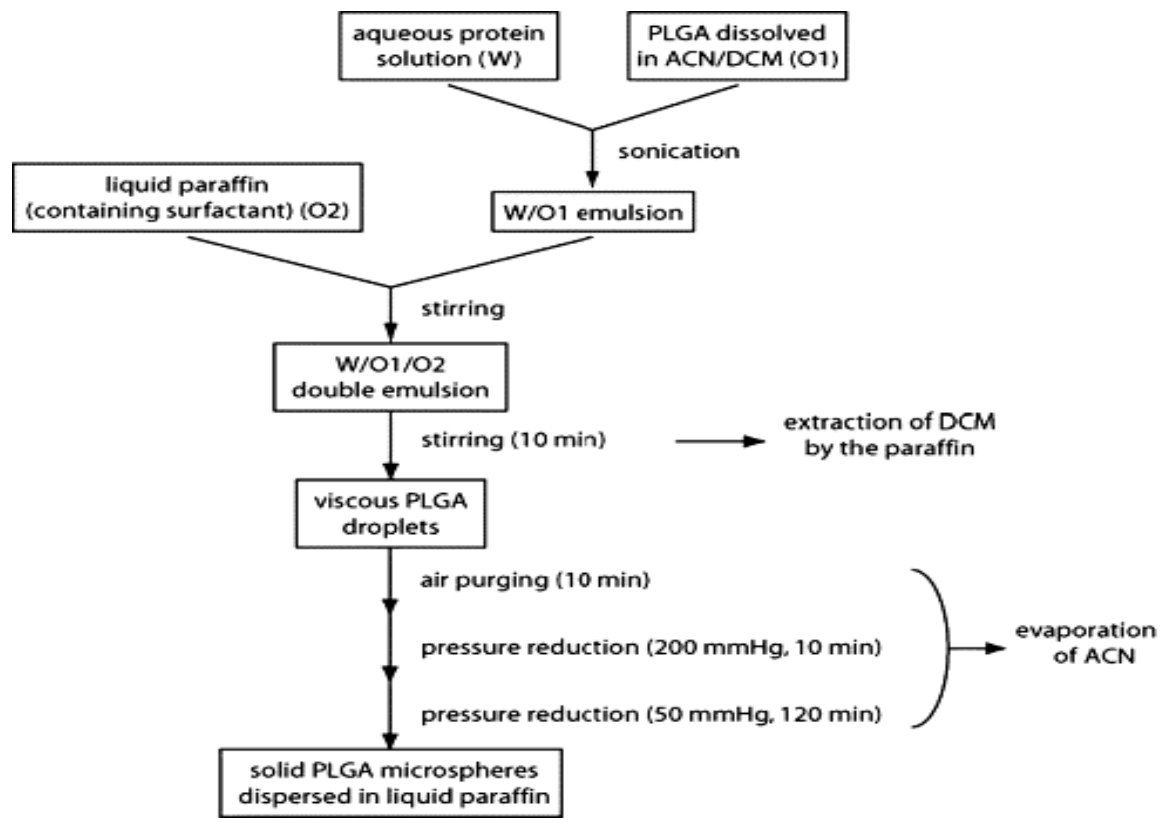

Fig. 5: Solvent extraction

\section{Phase separation co-acervation technique}

Phase separation method is mainly designed for preparing the reservoir type of the system. This method is used to encapsulate water soluble drugs e. g. peptides, proteins and some of preparations having matrix type particular, when the drug is hydrophobic in nature e. g. steroids. The process is based on the principal of decreasing the solubility of the polymer in the organic phase to affect the formation of the polymer rich phase called the coacervates. The coacervation can be brought about by the addition of the third component to the system which results in the formation of the two phases, one rich in polymer, while other not, i.e. supernant, depleted of the polymer. There are various methods which are effectively employed for coacervates phase separation.
The methods are based on the salt addition, on-solvent addition, addition of the incompatible polymer $[17,18]$.

\section{Solvent evaporation technique}

This is one of the earliest methods of microsphere manufacture. The polymer and drug must be soluble in an organic solvent, frequently methylene chloride.

The solution containing the polymer and the drug may be dispersed in an aqueous phase to form droplets. Continuous mixing and elevated temperatures may be employed to evaporate the more volatile organic solvent and leave the solid polymer-drug particles suspended in an aqueous medium. The particles are finally filtered from the suspension. 


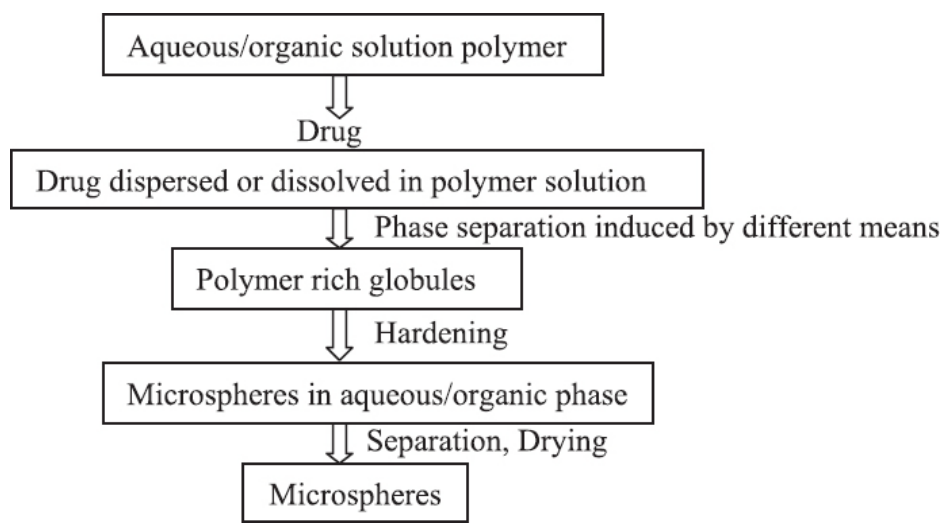

Fig. 6: Phase separation co-acervation technique

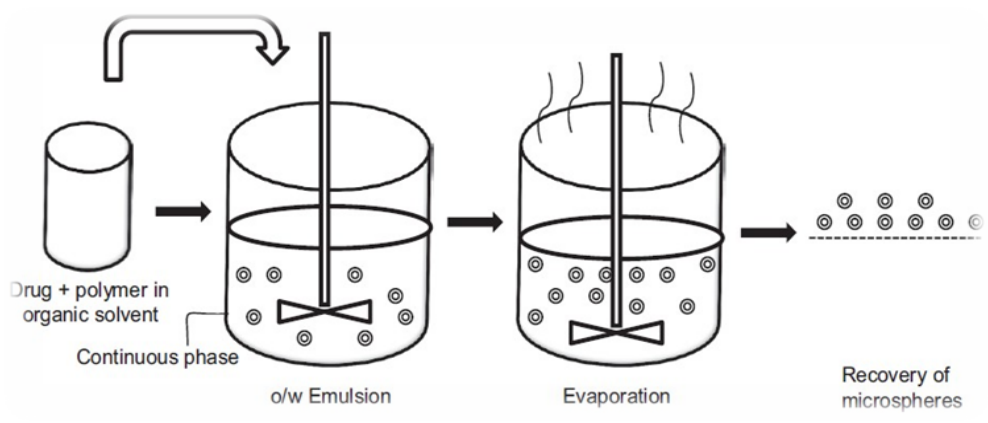

Fig. 7: Solvent evaporation technique

\section{Physicochemical evaluation}

\section{Particle size and shape}

Particle size can be determined by optical microscopy with the help of calibrated eyepiece micrometer. The size of around 100 microspheres is measured and their average particle size is calculated

$$
\mathrm{D} \text { mean }=\sum \mathrm{nd} / \sum \mathrm{n}
$$

Where, $n=$ number of microspheres checked; $d=$ Mean size

\section{Density determination}

The density of microspheres can be measured by using a multi volume pycnometer. Accurately weighed sample in a cup is placed into the multi volume pycnometer. Helium is introduced at a constant pressure in the chamber and allowed to expand. This expansion results in decrease in pressure within the chamber. Two consecutive readings of reduction in pressure at different initial pressure are noted. From two pressure readings the volume and hence the density of microsphere carriers is determined.

\section{Isoelectric point}

The isoelectric point can be measured by using micro electrophoresis apparatus by measuring electrophoretic mobility of microspheres. The mean velocity at different $\mathrm{pH}$ value from $3-10$ is calculated by measuring the time of particle movement over a distance of $1 \mathrm{~nm}$.

\section{Angle of contact}

The angle of repose $\emptyset$ of microspheres, which measures the resistance to particle flow is calculated as where, $2 \mathrm{~h} / \mathrm{d}$ is the surface area of free standing height of microspheres heap that is formed after making microspheres flow from the glass funnel

\section{Electron spectroscopy for chemical analysis}

The surface chemistry of microspheres can be determined by using electron spectroscopy for chemical analysis (ESCA). ESCA provides a means for the determination of atomic composition of the surface. The spectra obtained using ESCA can be used to determine the surface degradation of biodegradable microspheres.

\section{Fourier transform infrared spectroscopy}

Drug polymer interaction and degradation of microspheres can be assessed by FTIR.

\section{Drug entrapment efficiency}

Weighed amount of microsphere are taken and crushed. Then dissolved in buffer solution with the help of stirrer and filtered. The filtrate is assayed by UV spectrophotometer at particular wavelength by using calibration curve.

$$
\text { Drug Entrapment efficiency }=\frac{\text { Actual weight of microspheres }}{\text { Theoretical weight of drug and polymer }} \times 100
$$

\section{Percentage yield}

It is calculated as the weight of microspheres obtained from each batch divided by total weight of drug and polymer used to prepare that batch multiplied by 100

\section{Swelling index}

It is determined by measuring the extent of swelling of microspheres in a particular solvent. The equilibrium swelling degree of microspheres is determined by swelling of $5 \mathrm{mg}$ of dried microspheres poured in $5 \mathrm{ml}$ of buffer solution overnight in a measuring cylinder. It is calculated by given formula.

$$
\text { Swelling index }=\frac{\text { Mass of swollen microsphere }- \text { Mass of dried microspheres }}{\text { Mass of dried microsphares }} \times 100
$$

\section{In vitro methods}

This method allows the determination of release characteristics and permeability of a drug through membrane. In vitro method is employed as a quality control procedure in pharmaceutical production and in product development etc. Sensible and 
reproducible release data derived from physically, chemically and hydro dynamically defined conditions are necessary.

\section{Beaker method}

In this method Dosage form is made to adhere at the bottom of beaker containing the medium and stirred uniformly using overhead stirrer. Volume of the medium used in the literature for the studies varies from $50-500 \mathrm{ml}$ and the stirrer speed from 60-300 rpm.

\section{Interface diffusion method}

This method was developed by Dearden and Tomlinson. It consists of four compartments. Compartment A represents the oral cavity, and initially containing an appropriate concentration of drug in buffer. The compartment $\mathrm{B}$ representing the buccal membrane, containing 1-octanol, and compartment $\mathrm{C}$ representing body fluids, containing $0.2 \mathrm{M} \mathrm{HCl}$. The compartment $\mathrm{D}$ represents protein binding, also containing 1-octanol. Before use, the aqueous phase and 1-octanol are saturated with each other. Samples are withdrawn and returned to compartment $\mathrm{A}$ with a syringe.

\section{Modified keshary chien cell method}

It utilizes specialized laboratory designed apparatus. It comprises of a Keshary Chien cell containing distilled water $(50 \mathrm{ml})$ at $37^{\circ} \mathrm{C}$ as dissolution medium. TMDDS (Trans Membrane Drug Delivery System) is placed in a glass tube fitted with a $10 \#$ sieve at the bottom which reciprocates in the medium at 30 strokes per minute.

\section{Dissolution apparatus method}

Standard USP or BP dissolution apparatus have been used to study in vitro release profiles using both rotating elements Paddle and basket. Dissolution medium used for the study varies from 100-500 $\mathrm{ml}$ and speed of rotation from $50-100 \mathrm{rpm}$.

\section{In vivo method}

Method for studying the permeability of intact mucosa comprises of technique that gives the biological response of the organism locally or systemically and those that involve direct local measurement of uptake or accumulation of penetrate at their surface. The most widely used methods of in vivo studies include using animal models, buccal absorption tests.

\section{Animal models}

It is used mainly for the screening of series of compounds, investigating the mechanisms and evaluating a set of formulations. Animal model such as dogs, rats, pigs and sheep etc. are reported. Generally the procedure involves anesthetizing the animal followed by administration of dosage form, withdrawing blood at different time intervals and analyzing.

\section{Buccal absorption test}

It is most suitable and reliable method for measuring the extent of drug loss from human oral cavity for single and multi-component mixtures of drugs. The test has been successfully used to investigate the relative importance of drug structure, contact time, initial drug concentration and $\mathrm{pH}$ of solution while drug is held in oral cavity. The test is carried to measure the kinetics of the drug absorption by swirling a $25 \mathrm{ml}$ sample of the test solution for $15 \mathrm{~min}$ by human volunteers followed by the expulsion of the solution. The amount of the drug remaining in the expelled volume is then determined to assess the amount of drug absorbed.

\section{In vitro/in vivo correlation}

Correlations between in vitro dissolution rates and the rate and extent of availability as determined by blood concentration and or urinary excretion of drug or metabolites are referred to as "in vitroin vivo correlation". Such correlations allow one to develop product specifications with availability.

\section{Applications of microspheres}

\section{Microspheres in vaccine delivery}

The prerequisite of a vaccine is protection against the microorganism or its toxic product. An ideal vaccine must fulfill the requirement of efficacy, safety, convenience in application and cost. The aspect of safety and minimization of adverse reactions a complex issue. The aspect of safety and the degree of the production of antibody responses are closely related to mode of application. Biodegradable delivery systems for vaccines that are given by parenteral route may overcome the shortcoming of the conventional vaccines. The interest in parenteral (subcutaneous, intramuscular, intradermal) carrier lies since they offer specific advantages including:

- Improved antigen city by adjuvant action

- Modulation of antigen release

- Stabilization of antigen.

\section{Targeting using micro particulate carriers}

The concept of targeting, i.e. site specific drug delivery is a wellestablished dogma, which is gaining full attention. The therapeutic efficacy of the drug relies on its access and specific interaction with its candidate receptors. The ability to leave the pool in reproducible, efficient and specific manner is enter to drug action mediated by use of a carrier system.

\section{Monoclonal antibodies facilitated microspheres targeting}

Monoclonal antibodies targeting microspheres are immune microspheres. This targeting is used to achieve selective targeting to specific sites. Monoclonal antibodies are extremely specific molecules. This extreme specificity of monoclonal antibodies (Mabs) can be utilized to target microspheres loaded bioactive molecules to selected sites. Mabs can be directly attached to the microspheres by means of covalent coupling. The free aldehyde groups, amino groups or hydroxyl groups on the surface of the microspheres can be linked to the antibodies.([15,16]) Maps can be attached to microspheres by any of following methods:

- Nonspecific adsorption and specific adsorption

- $\quad$ Direct coupling

- Coupling via reagent

\section{Imaging}

The microspheres have been extensively studied and used for the targeting purposes. Various cells, cell lines, tissues and organs can be imaged using radio labelled microspheres. The particle size range of microspheres isa vital factor in determining the imaging of particular sites. The particles injected intravenously apart from the portal vein will become entrapped in the capillary bed of the lungs. This phenomenon is abused for the scintigraphicimaging of the tumor masses in lungs using labelled human serum albumin microspheres $[19,20]$.

\section{Topical porous microspheres}

Microsponges are porous microspheres having myriad of interconnected voids of particle size range 5-300 $\mu \mathrm{m}$. These microsponges having capacity to entrap wide range of active ingredients such as emollients, fragrances, essential oils etc., are used as the topical carries system further, these porous microspheres with active ingredients can be incorporated into formulations such as creams, lotions and powders. Microsponges consist of non-collapsible structures with porous surface through which active ingredients are released in a controlled manner.

\section{Nasal drug delivery}

Intranasal (IN) administration has many theoretical and practical advantages for the local and systemic delivery of a diverse therapeutic compound. IN delivery is needle free, non-invasive, and essentially painless, does not require sterile preparation, and can be self-administered. The large surface area of the nasal mucosa originated from the presence of a large number of microvilli, a porous endothelial membrane, and a highly vascularized epithelium serves a rapid onset of therapeutic effect. It is describes various systems, devices, formulations, and methods of delivery of drugs to the nose or nasal cavity. Depending on the therapeutic intent, 
intranasal drugs may be targeted for local treatment or systemic action. In nasal drug delivery, coupling of bioadhesive properties to microspheres is of great importance because of additional advantages: efficient absorption and enhanced bioavailability of the drug, a much more intimate contact with the mucus layer and reduction in frequency of drug administration due to the reduction in mucociliary clearance of drug delivery system adhering to nasal mucosa.

\section{Gastroretentive controlled delivery system}

In which Floating systems are low-density systems that have float over the gastric contents and remain in the stomach for a prolonged period than conventional dosage forms. Gastric emptying of dosage form is extremely variable process and ability to control the emptying time is valuable asset for dosage forms, there are several difficulties are faced in designing controlled released systems for better absorption and enhanced the bioavailability. While the system floats over the gastric contents, the drug is released slowly at the desired rate, which results in increased gastro-retention time and reduces fluctuation in plasma drug concentration. Several polymers are used for gastro retentive controlled delivery system such are Cellulose acetate, Chitosan, Eudragit, Acrycoat, Methocil, Polyacrylates, Polyvinyl acetate, carbopol, Agar, Polyethylene oxide, Polycarbonates, Acrylic resins and Polyethylene oxide etc.

\section{Implantable devices}

Microencapsulation has also been used medically for the encapsulation of live cells and vaccines. Biocompatibility can be improved by the encapsulation of artificial cells and biomolecules such as peptides, proteins, and hormones, which can prevent unwanted immunological reactions that would lead to inactivation or rejection. Microspheres are used for isolating materialsuntil their activity is needed. The biotechnology industry employs microspheres to contain organisms and their recombinant products to aid in the isolation of this products.

\section{Pharmaceutical applications}

A number of pharmaceutical microencapsulated products are currently on the market, such as aspirin, theophylline and its derivatives, vitamins, pancrelipase, antihypertensives, potassium chloride, progesterone, and contraceptive hormone combinations. Microencapsulated KCL is used to prevent gastrointestinal complications associated with potassium chloride. The dispersibility of the microcapsules and the controlled release of the ions minimize the possibility of local high salt concentrationswhich could result in ulceration, hemorrhage, or perforation. Microspheres have also found potential applications as injection, or inhalation products. The number of commercially available products does not reflect the amount of research that has been carried out in this area, nor the benefits that can be achieved using this technology. Economic considerations have been a key factor in determining the number of pharmaceutical microencapsulated products. Most encapsulation processes are expensive and require significant capital investment for equipment. An exception is pan or sprays coating and spray drying, since the necessary equipment may already be available within the company. An additional expense is due to the fact that most microencapsulation processes are patent protected.

\section{Other applications}

Fluorescent microspheres can be used for membrane based technology for flow cytometry, cell biology, microbiology, Fluorescent Linked Immuno-Sorbent Assay. Yttrium can be used for primary treatment of hepatocellular carcinoma and also used for pre transplant management of HCC with promising results. Applications of microencapsulation in other industries are numerous. The best known microencapsulated products are carbonless copying paper, photosensitive paper, microencapsulated fragrances, such as "scentstrips" (also known as "snap-n-burst"), and microencapsulated aromas ("scratch-n-sniff"). All of these products are usually prepared by gelatin-acacia complex coacervation. Scratch-n-sniff has been used in children's books and food and cosmetic aroma advertising. Microcapsules are also extensively used as diagnostics, for example, temperature-sensitive microcapsules for thermo graphic detection of tumors. In the biotechnology industry microencapsulated microbial cells are being used for the production of recombinant proteins and peptides.

\section{CONCLUSION}

Microspheres are better choice of drug delivery system than many other types of drug delivery system. In future by combining various other strategies, microspheres will find the central and significant place in novel drug delivery, particularly in diseased cell sorting, diagnostics, gene and genetic materials, safe, targeted, specific and effective in vitro delivery and supplements as miniature version of diseased organ and tissues in the body. Microspheres offer several improvements over existing technologies. These have emerged as an exciting new platform for biologists to adopt these techniques in the investigation of biomolecules interactions and cellular processes. In recent years there have been increasingnumbers of studies in which microspheres have been used in more diverse applications and it is evident that the range of potential applications is enormous. In addition, microspheres have been labeled with a variety of $\beta$ and emitting radionuclide such as $131 \mathrm{I}, 99 \mathrm{mTc}, 113 \mathrm{mIn}$ or $51 \mathrm{Cr}$. Such products have been used to scan the heart, brain, liver and gastro intestinal tracts and in a pulmonary perfusion and inhalation studies. Microsphere is a short term but it is having wide applications in drug delivery systems to get desire biological activity. By combining various strategies, microspheres will find central place in novel drug delivery system mainly particularly in cell sorting, diagnostics and Genetic engineering. From the study it is proved that Microspheres act as effective carriers for the novel drug delivery system.

\section{AUTHORS CONTRIBUTIONS}

All the author have contributed equally

\section{CONFLICT OF INTERESTS}

\section{Declare none}

\section{REFERENCES}

1. Freitas S, Merkle HP, Gander B. Microencapsulation by solvent Extraction/Evaporation: reviewing the state of the art of microsphere preparation process technology. J Controlled Release 2004;102:313-32.

2. Sahil K, Akanksha M, Premjeet S, Bilandi A, Kapoor B, Microsphere: a review. Int J Res Pharm Chem 2011;1:2231781.

3. Rajput S, Agrawal P, Pathak A, Shrivasatava N, Baghe SS, Baghe RS. A review on microspheres: methods of preparation and evaluation. World J Pharm Pharm Sci 2012;1:422-38.

4. Meghna KS, Krishna MP, Giridas S, Sreelakshmi C, Vijayakumar B. Microsphere a drug delivery system-a review. Int J Novel Trends Pharm Sci 2017;7:109-18.

5. Kumar A, Mahajan S, Bhandari N, Microspheres: a review. World J Pharm Pharm Sci 2017;6:724-40.

6. Vikrant KN, Gudsoorkar VR, Hiremath SN, Dolas RT, Kashid VA. Microspheres-a novel drug delivery system: an overview. Int J Pharm Chem Sci 2012;1:113-28.

7. Gholap SB, Banarjee SK, Gaikwad DD, Jadhav SL, Thorat RM. Hollow microsphere: a review. Int J Pharm Sci Rev Res 2010;1:10-5.

8. Agusundaram M, Madhu SC. Microsphere, as a novel drug delivery system a review. Int J ChemTech Res 2009;1:526-34.

9. Sudha MT, Naveen KK. At preparation and evaluation of ethyl cellulose microspheres of ibuprofen for sustained drug delivery. Int J Pharma Res Dev 2010;2:120-1.

10. Thanoo BC, Sunny MC, Jayakrishnan A. Cross-linked chitosan microspheres: preparation and evaluation as a matrix for the controlled release of pharmaceuticals. J Pharm Pharmacol 1992;44:283-6.

11. Parmar H, Bakliwal V. Different method of evaluation of mucoadhesive microsphere. Int J Appl Biol Pharm Technol 2010;1:1164-5.

12. Kalyan S, Sharma PK. Recent advancement in chitosan best formulation and its pharmaceutical application. Pelagia Res Library 2010;1:195-210. 
13. Orienti I, Aiedeh K, Gianasi E, Bertasi V, Zecchi V. Indomethacin loaded chitosan microspheres correlation between the erosionprocess and release kinetics. J Microencapsul 1996;13:463-72.

14. Nair R, Reddy B. Application of chitosan microspheres as drug carriers: a review. J Pharm Sci Res 2009;1:1-12.

15. Kadam NR, Suvarna V. Microsphere a brief review. Asian J Biomed Pharm Sci 2015;5:13-9.

16. Kataria S, Middha A. Microsphere a review. Int J Res Pharm Chem 2011;1:2237-781.
17. Alagusundaram M, Madhu SC. Microsphere as a novel drug delivery system a review. Int J Chem Tech Res 2009;1:526-34.

18. Thummar AV, Kyada CR, Kalyanvat R, Shreevastva B. A review on mucoadhesive microspheres as a novel drug delivery system. Int J Pharm Scholar 2013;2:188-200.

19. Pradeesh TS, Sunny MC, Varma HK, Ramesh P. Preparation of microstructured hydroxyapatite microspheres using oil in water emulsions. Bull Materials Sci 2005;28:383-90.

20. Jayaprakash S, Halith SM, Mohamed Firthouse PU, Kulaturanpillai K. Preparation and evaluation of biodegradable microspheres of methotrexate. Asian J Pharm 2009;3:26-46. 